\title{
Peculiarities of magnetocaloric effect in manganites connected with magnetic heterogeneous state
}

\author{
L. I. Koroleva, A. S. Morozov \\ M.V. Lomonosov Moscow State University, 119992 Leninskie Gory, Moscow, Russia \\ Email address: \\ Koroleva@phys.msu.ru (L. Koroleva)
}

\section{To cite this article:}

L. I. Koroleva, A. S. Morozov. Peculiarities of Magnetocaloric Effect in Manganites Connected with Magnetic Heterogeneous State, American Journal of Modern Physics. Vol. 2, No. 2, 2013, pp. 61-67. doi: 10.11648/j.ajmp.20130202.15

\begin{abstract}
Magnetocaloric effect (MCE) or $\Delta T$-effect was studied in $\mathrm{La}_{1-\mathrm{x}} \mathrm{Sr}_{\mathrm{x}} \mathrm{MnO}_{3}, \mathrm{Sm}_{0.55} \mathrm{Sr}_{0.45} \mathrm{MnO}_{3}$ and $\mathrm{PrBaMn}_{2} \mathrm{O}_{6}$ manganites. It has been found that the maximum value of MCE, measured by direct method, is far less than obtainable by computation from the change of the magnetic entropy in Curie temperature $T_{C}$. This phenomenon is explained by presence in them of the magnetic two-phase ferromagnetic (F) - antiferromagnetic (AF) state. So in $\mathrm{La}_{1-\mathrm{x}} \mathrm{Sr}_{\mathrm{x}} \mathrm{MnO}_{3}$ the negative contribution from AF portion of sample lowers MCE and displacesmaximum on $\Delta T(T)$-curve to the higher temperature than $T_{C}$ on 20-40 K. Maximum on $\Delta T(T)$ curve of $\mathrm{Sm}_{0.55} \mathrm{Sr}_{0.45} \mathrm{MnO}_{3}$ is disposed near $T_{C}=134 \mathrm{~K}$ in the cooling in the air single-crystal and ceramic samples. The cooling inoxygen of single-crystal,restored the Mn-O-Mn broken connections, increases the volume of clusters with CE-type of AF order, results to that maximum on $\Delta T(T)$ curve is disposed at Neel temperature $T_{N}$ of this phase $(243 \mathrm{~K})$. Magnetic field, applied to sample during $\Delta T$-measurement, transforms AF clusters into F state and theboth types of clusters decompose at $T_{N}$. The $\mathrm{PrBaMn}_{2} \mathrm{O}_{6}$ manganite has two phase transactions: paramagnetic $-\mathrm{F}$ at $T_{C}=295 \mathrm{~K}$ and $\mathrm{F}-\mathrm{AF}$ at $T_{N}=231 \mathrm{~K}$. Phase with spontaneous magnetization obtains the understated magnetic moment. Curve $\Delta T(T)$ has the wide maximum near $T_{C}$ and the sharp minimum near $T_{N}$ with the small $\Delta T$-values in both extremes: $0,13 \mathrm{~K}$ and - $0,2 \mathrm{~K}$ respectively. This is connected with presence of AF interactions in $\mathrm{F}$ phase and $\mathrm{F}$ interactions in AF phase.
\end{abstract}

Keywords: Magnetocaloric Effect, Nagnetization, Paramagnetic Susceptibility, Magnetic Two-Phase State, Manganite

\section{Introduction}

The temperature change induced in magnetic material during an adiabatic magnetic field change is the magnetocaloric effect (MCE). MCE provides the physical basis of magnetic refrigeration technology.Application of magnetic cooling will decrease the global consumption of energy and consumer chemistry, which is necessary for weakening the decrease in the ozone layer thickness near the Earth, and can be an alternative to the gas compression in refrigerating engineering in the near future. Therefore, researchers pay particular attention to searching for and studying the materials having a high MCE at near room temperatures. According to classical thermodynamics $\mathrm{MCE}$ is estimated from the formula:

$$
\Delta \mathrm{T}_{\mathrm{th}}=\frac{\mathrm{T}}{\mathrm{C}_{\mathrm{H}}(\mathrm{T})} \Delta \mathrm{S}_{\mathrm{M}}(\mathrm{T}),
$$

where $C_{H}$ is specific heat at a constant field and $\Delta S_{M}(T)$ is the magnetic entropy change of the magnet, when a field is applied adiabatically:

$$
\Delta \mathrm{S}_{\mathrm{M}}=\int_{0}^{\mathrm{H}}\left(\frac{\mathrm{dM}}{\mathrm{dT}}\right)_{\mathrm{H}} \mathrm{dH} .
$$

Here $M$ is magnetization. The large change of magnetic entropy in the Curie point was observed in manganites in works [1-17]. In some compounds this change is more, than for Gd, for which the MCE value reaches $5 \mathrm{~K}$ in the field $2 \mathrm{~T}[4,5]$. Therefore, manganites should have a large MCE.

But magnetic two-phase ferro-antiferromagnetic (AF) state, caused by strong $s-d$ exchange, exists in manganites [18 - 20]. It should have an impact on the MCE, as MCE for AF's is negative and for ferromagnets (F's) the one is positive properly from relations $(1,2)$. In [21], we showed that a weak exchange coupling between the $\mathrm{F}$ and AF parts of a sample exists in this case. Therefore MCE can be presented in the form:

$$
\Delta \mathrm{T}=\Delta \mathrm{T}_{\mathrm{F}}+\Delta \mathrm{T}_{\mathrm{AF}}
$$

where $\Delta T_{\mathrm{F}}$ and $\Delta T_{\mathrm{AF}}$ are the MCEs of the $\mathrm{F}$ and $\mathrm{AF}$ parts of the sample, respectively. As it is seen from Eqs. (1) and (2), $\Delta T_{\mathrm{F}}$ is positive and $\Delta T_{\mathrm{AF}}$ is negative. In this case, 
the MCE estimated from the sharp decrease in the magnetization near the Curie temperature (see Eq. (1)) corresponds only to the F part of the sample; hence, it is overestimated because of the fact that negative contribution $\Delta T_{\mathrm{AF}}$ is not taken into account. Therefore, the magnitude and sign of MCE in manganites can only be determined by direct measurements of this effect.

The purpose of this work is to use a direct method to study MCE.MCE and magnetization were studied in compounds: $\mathrm{La}_{1-\mathrm{x}} \mathrm{Sr}_{\mathrm{x}} \mathrm{MnO}_{3}, \mathrm{Sm}_{0.55} \mathrm{Sr}_{0.45} \mathrm{MnO}_{3}$ and $\mathrm{PrBaMn}_{2} \mathrm{O}_{6}$ belonged to three classes of manganites: $\mathrm{La}_{1-\mathrm{x}} \mathrm{A}_{\mathrm{x}} \mathrm{MnO}_{3}$ (A $=\mathrm{Ca}, \mathrm{Sr}, \mathrm{Ba}), \mathrm{Sm}_{0,5+\mathrm{x}} \mathrm{Sr}_{0.5-\mathrm{x}} \mathrm{MnO}_{3}(0,05 \leq x \leq 0.10)$ and $\mathrm{ReBaMn}_{2} \mathrm{O}_{6}(\mathrm{Re}=\mathrm{Pr}, \mathrm{Nd}, \mathrm{Sm}, \mathrm{Eu}, \mathrm{Gd})$ with the layer crystalline structure. Our results have shown that values of MCE received by direct method $\left(\Delta T_{e x}\right)$ are well smaller than calculated from the magnetization change in T-region $\left(\Delta_{t h}\right)$.

\section{Experimental}

MCE was studied on $\mathrm{La}_{1-\mathrm{x}} \mathrm{Sr}_{\mathrm{x}} \mathrm{MnO}_{3}(x=0.1,0.125,0.3)$, $\mathrm{Sm}_{0.55} \mathrm{Sr}_{0.45} \mathrm{MnO}_{3}$ single crystals and $\mathrm{PrBaMn}_{2} \mathrm{O}_{6}$, $\mathrm{Sm}_{0.55} \mathrm{Sr}_{0.45} \mathrm{MnO}_{3}$ (C-sample) ceramics samples. The ceramic samples were prepared by standard ceramic techniques by A.R. Kaul and single crystals were grown by the floating-zone method by A.M. Balbashov. One single crystal $\mathrm{Sm}_{0.55} \mathrm{Sr}_{0.45} \mathrm{MnO}_{3}$ was annealed in air (A-sample) and other in oxygen atmosphere (O-sample). Using X-ray diffraction, the all samples were found to be pure singlephase perovskite with the orthorhombic Pnma structure. To achieve a high degree of crystallographic ordering between the $\mathrm{Pr}$ and $\mathrm{Ba}$ ions in the $\mathrm{PrBaMn}_{2} \mathrm{O}_{6}$ compound, they performed annealing in an oxygen atmosphere. The degree of ordering in the best samples reached $96 \%$,.

MCE was measured by direct method. Magnetic field $H$ for quasistatic measurement was ranged from 0 to 14.2 $\mathrm{kOe}$ and was created by an electromagnet. The equilibrium temperature $T$ of the sample was measured using copper-constantan thermocouple before and after the introduction of a magnetic field. The thermocouple joint was placed inside a sample.The MCE was determined as the difference between these two equilibrium temperatures. Miniminization of the heat losses was reached by high vacuum (not less than $10^{-5} \mathrm{~mm} \mathrm{Hg}$ ), heat screen around the sample and by usage of ultrathin detector wires. The experimental setup was tested with gadolinium sample of $99.99 \%$ purity.

The magnetization of the sample was also measured with SQUID and vibrating-sample magnetometers, Theparamagnetic susceptibility was measured by weighing with electromagnetic compensation.

\section{Magnetocaloric Effect in $\mathrm{La}_{(1-}$ ${ }_{\mathrm{x})} \mathrm{Sr}_{\mathrm{x}} \mathrm{MnO}_{3}$ System}

Figure 1 shows the temperature dependences of magnetization $M$ in a magnetic field of $8.2 \mathrm{kOe}$, and Figure 2 shows the temperature dependences of MCE $\Delta T_{\exp }(T)$ in the same magnetic field measured by the direct method $(a)$ and the $\Delta T_{t h}(T)$ calculated by Eq. (1) $\{b\}$ on $\mathrm{La}_{1}$
${ }_{x} \mathrm{Sr}_{x} \mathrm{MnO}_{3}(x=0.100,0.125,0.300)$ single- crystal samples. The values of $\Delta T_{t h}$ was computed from the formula (1) in which $\Delta S_{M}$ was computed with the help (2) from a magnetization, and $C_{H}$ was taken from [22]. The numerical integration in (1) was realized by Sympson method. It is shown in Figure 2 that on the all curves the maxima are observed, but the temperaturesof these maxima for each compound are differed: on experimental curves they on 20-30 K are higher, than on the curves computed from formula (1). It should be noted that curve $\Delta T_{e x}(T)$ has step in those regions temperatures, where is maximum on curve $\Delta T_{t h}(T)$. It is possible to explain these facts by the presence of the magnetic two-phase F-AF state in the sample. In this case the difference betweencurves $\Delta T_{e x}(T)$ and $\Delta T_{\text {th }}(T)$ reflects the contribution of AF part of the sample to the experimentally measured MCE, which is shown in Figure 3. This AF contribution has the shape characteristic of antiferromagnets [23]: it is negative in the temperature range of breaking an AF order, and MCE is positive above this range (which is characteristic of paramagnetic order). It reverses sign at Neel temperature $T_{N}$. It follows that the values of $T_{C}$ and $T_{N}$ are closely allied in investigated compounds. In this consideration we neglected by an entropy change of F/AF interface. However, in paper [21] we showed that the exchange interaction between $\mathrm{F}$ and AF regions is much less than the exchange inside these regions and consequently the transition region between them practically does not exist.

It is known thatthe $\mathrm{F}$ phase occupies almost the entire sample volume in the composition with $x=0.30$ at low temperatures, although the contribution of the AF part to $\Delta T_{\text {exp }}$ is high (see Fig. 3). This finding is likely to be related to the fact that the magnetic heterogeneity grows strongly at the $T_{\mathrm{C}}$-region in this composition. For example,the data of neutron diffraction [24], EPR [25], absorption spectra [26] and other studies reviewed in [19] point to the existence of a mixed $\mathrm{F}-\mathrm{AF}$ state near $T_{\mathrm{C}}$ in compositions with $x \leq 0.3$.

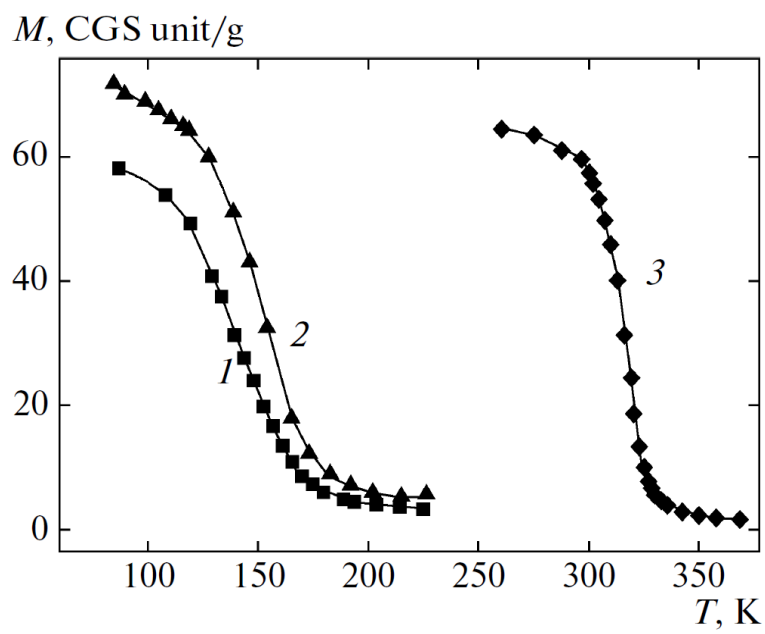

Figure 1. Temperature dependences of the magnetization of singlecrystal $\mathrm{La}_{1-x} \mathrm{Sr}_{x} \mathrm{MnO}_{3}$ samples in a magnetic field of $8.2 \mathrm{kOe}$ at $x=$ (1) 0.100 , (2) 0.125 and (3) 0.300 . 

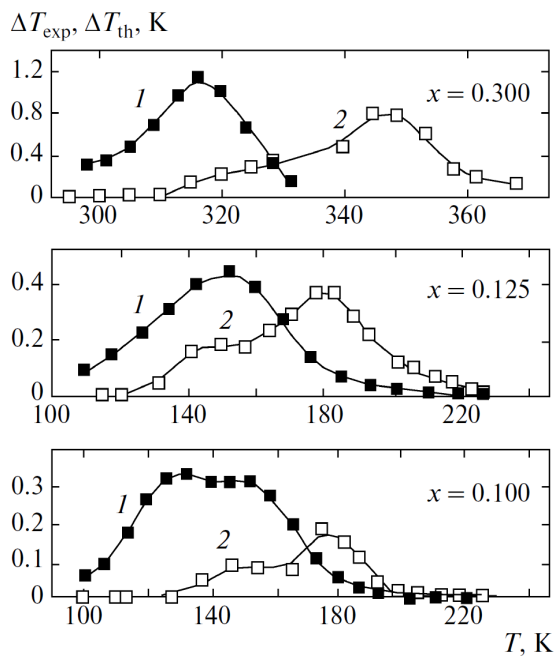

Figure 2. Temperature dependences of MCE obtained by a direct method (UTexp (T), curves 2) and calculated by Eq. (1) (UTth(T), curves 1) in a field of $8.2 \mathrm{kOe}$ for single-crystal $\mathrm{La}_{1-x} \mathrm{Sr}_{x} \mathrm{MnO} \mathrm{O}_{3}$ samples.
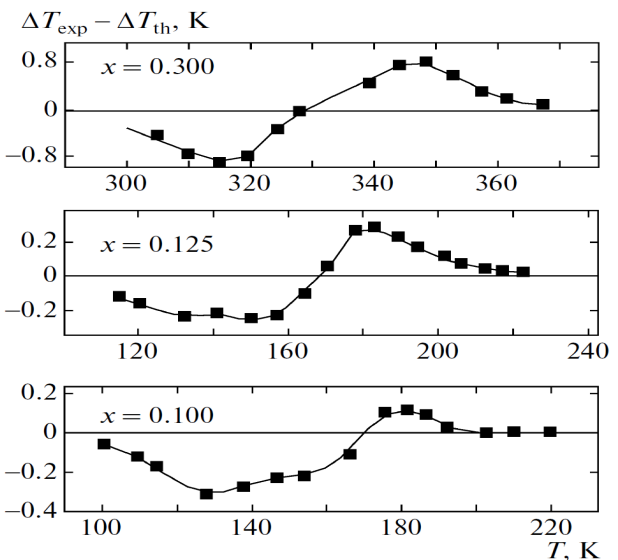

Figure 3. MCE due to a break in the AFM order in an AFM phase in a field of $8.2 \mathrm{kOe}$ for single-crystal $\mathrm{La}_{1-x} \mathrm{Sr}_{x} \mathrm{MnO}_{3}$ samples.
The Curie temperatures of the compositions under study were determined as the temperatures of the maxima in the $(d M / d T)(T)$ curves measured in a low magnetic field of $100 \mathrm{Oe}$. They were found to be $140 \mathrm{~K}, 150 \mathrm{~K}$, and 315 $\mathrm{K}$ for the compositions with $x=0.100,0.125,0.30$ respectively. It should benoted that the concept of the Curie and Néel temperatures is rather conventional in magnetically heterogeneous magnets, which also include the compositions under study. Here, these are the temperatures of breaking a magnetic order in the $\mathrm{F}$ and $\mathrm{AF}$ phases of a sample. As is seen from a comparison of Figures 2 and 3, the negative MCE induced by the AF part of a sample decreases the maximum value of $\Delta T \exp (T)$ curve as compared to the value that could be if the sample contained only an $\mathrm{F}$ phase and shifts the maximum in the $\Delta T \exp (T)$ curve above TC. This maximum in F metals is usually located at the Curie temperature [27].

Thus, with the help of magnetic two-phase $\mathrm{F}-\mathrm{AF}$ state presence in sample it is possible to explain anomaly behavior of $\Delta T_{e x}(T)$ in these compounds.

\section{Magnetocaloric Effect in $\mathrm{Sm}_{0.55} \mathrm{Sr}_{0.45} \mathrm{MnO}_{3}$}

Recent neutron and electron diffraction studies of $\mathrm{Sm}_{0.5+\mathrm{x}} \mathrm{Sr}_{0.5-\mathrm{x}} \mathrm{MnO}_{3}[28,29]$ have shown that a magnetic inhomogeneous state consisting of F clusters, A-type AF clusters and CE-type AF clusters is presented in them, Both $\mathrm{F}$ and A-type AF clusters break down in $T_{C}$-region and CE-type AF clusters remain at $T>T_{C}$. The $T_{C}$-values of A-, O- and C-samples, determined by us from the AC initial magnetic susceptibility measurements, are $130 \mathrm{~K}$, $134 \mathrm{~K}$ and $142 \mathrm{~K}$ respectively (See Table I).

Table 1. Magnetocaloric effect and magnetic characteristics of $\mathrm{Sm}_{0.55} \mathrm{Sr}_{0.45} \mathrm{MnO}_{3}$ and $\mathrm{PrBaMn}{ }_{2} \mathrm{O}_{6}$

\begin{tabular}{|c|c|c|c|c|c|c|c|}
\hline Composition & $T_{C}\left(T_{N}\right), \mathbf{K}$ & $\theta, \mathbf{K}$ & $\Delta T_{\text {exp }}, \mathbf{K}$ & $\begin{array}{l}T_{\max }\left(T_{\min }\right) \\
(\exp .), \mathbf{K}\end{array}$ & $\Delta S_{M}, \mathbf{J} / \mathrm{kg} \mathrm{K}$ & $\Delta T_{t h}, \mathbf{K}$ & $T_{\max }$ (th.), $\mathbf{K}$ \\
\hline $\begin{array}{l}\mathrm{Sm}_{0.55} \mathrm{Sr}_{0.45} \mathrm{MnO}_{3} \\
\text { sample A }\end{array}$ & 130 & 225 & 0.75 & 133 & 15.93 & 6.7 & 138 \\
\hline $\begin{array}{l}\mathrm{Sm}_{0.55} \mathrm{Sr}_{0.45} \mathrm{MnO}_{3} \\
\text { sample O }\end{array}$ & 134 & 270 & 0.40 & 243 & 12.62 & 5.3 & 144 \\
\hline $\begin{array}{l}\mathrm{Sm}_{0.55} \mathrm{Sr}_{0.45} \mathrm{MnO}_{3} \\
\text { sample C }\end{array}$ & 142 & 230 & 0.40 & 142 & 8.98 & 3.8 & 146 \\
\hline $\begin{array}{l}\mathrm{PrBaMn}_{2} \mathrm{O}_{6} \\
\text { polycrystal }\end{array}$ & (231) & 297 & -0.20 & $(236)$ & -5.06 & $-5.9^{*}$ & 234 \\
\hline
\end{tabular}

* When calculating $\Delta T$ th, we borrowed the specific heat from [30].

Figure 4 a,b,c shows the temperature and field dependences of the magnetization $M$ in high magnetic fields (up to $70 \mathrm{kOe}$ ) for sample $\mathrm{O}$, and similar curves were recorded for samples A and C. These measurements were carried out on two samples cut from single crystals in the form of the same parallelepipeds. The longest edge was parallel to the $c$ axis or the $a b$ plane of orthorhombic structure and parallel to a magnetic field during the magnetization measurements. The $M(T)$ and $M(H)$ curves of both parallelepipeds are almost the same, which indicates the absence of a magnetic anisotropy in these crystals.. The transition from the spontaneous magnetization to the paramagnetic phase is strongly smeared (see Figure. 4a). As it have been noticed above, this is associated with the presence of three types of magnetic clusters in the sample. The magnetization isotherms first linearly increase above the Curie point (Figure. 4b) due to the magnetization of AF clusters and then increases sharply in the range $H c_{1} \leq H \leq H c_{2}$ due to the transition of CE- type AF clusters into the F state. The field dependence of the magnetization becomes linear at $T$ 
$\approx 240 \mathrm{~K}$ (Figure $4 \mathrm{c}$ ), which can be considered as the temperature of breaking the $\mathrm{CE}$ type $\mathrm{AF}$ order $\left(T_{\mathrm{NCE}}\right)$ and charge ordering in these clusters. When the latter is broken, the crystal lattice changes substantially; i.e., this is an analog of a first order phase transition. The Figure $5 \mathrm{~d}$ shows the temperature dependence of inverse paramagnetic susceptibility $1 / \chi$ for all studied samples The shown $\{1 / \chi\}(T)$ dependence obeys the Curie-Weiss law with paramagnetic Curie temperatures $(\Theta)$ of 225,270 , and 230 $\mathrm{K}$ for samples $\mathrm{A}, \mathrm{O}$, and $\mathrm{C}$ respectively.
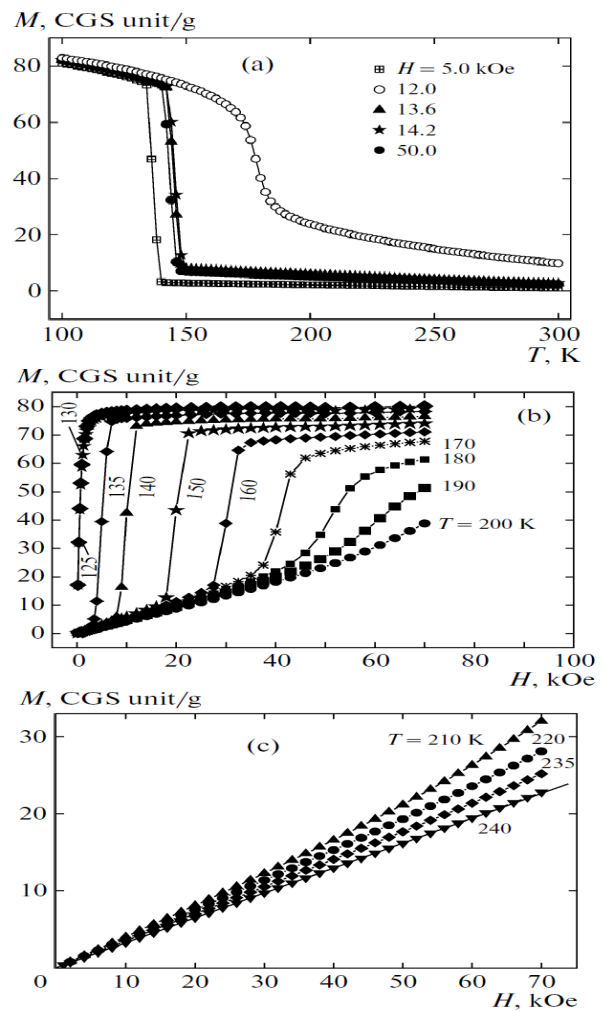

Figure 4. $\mathrm{La}_{1-\mathrm{x}} \mathrm{Sr}_{x} \mathrm{MnO}_{3}$ single-crystal sample O. Magnetization vs. (a) temperature and (b), (c) magnetic field at various temperatures.

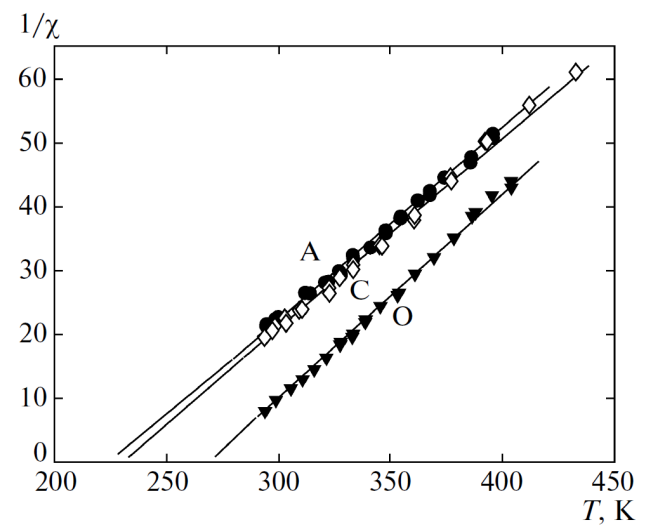

Figure 5. Temperature dependences of the inverse paramagnetic susceptibility of $\mathrm{La}_{1-x} \mathrm{Sr}_{x} \mathrm{MnO}_{3}$ for single-crystal

Figure 6 shows the dependence $\Delta T_{e x}(T)$ in magnetic field $14.2 \mathrm{kOe}$ of A-, C- and O-samples. As we can see from Figure 6a the $\Delta T_{e x}(T)$ curve of A-sample has maximum at $T_{\max }=143.3 \mathrm{~K}$ and minimum at $T_{\min }=120 \mathrm{~K}$ with $\Delta T_{e x}=0.8 \mathrm{~K}$ in maximum and $\Delta T_{e x}=-0.1 \mathrm{~K}$ in minimum. The maximum on the $\Delta T_{e x}(T)$ curve is observed too in the O-sample at $T_{\max }=244 \mathrm{~K}$ (Figure $6 \mathrm{~b}$ ) and the C-sample at $T_{\max }=143 \mathrm{~K}$ (Fugure $6 \mathrm{c}$ ). In these maxima MCE values are $0.41 \mathrm{~K}$ and $0.4 \mathrm{~K}$ respectively.
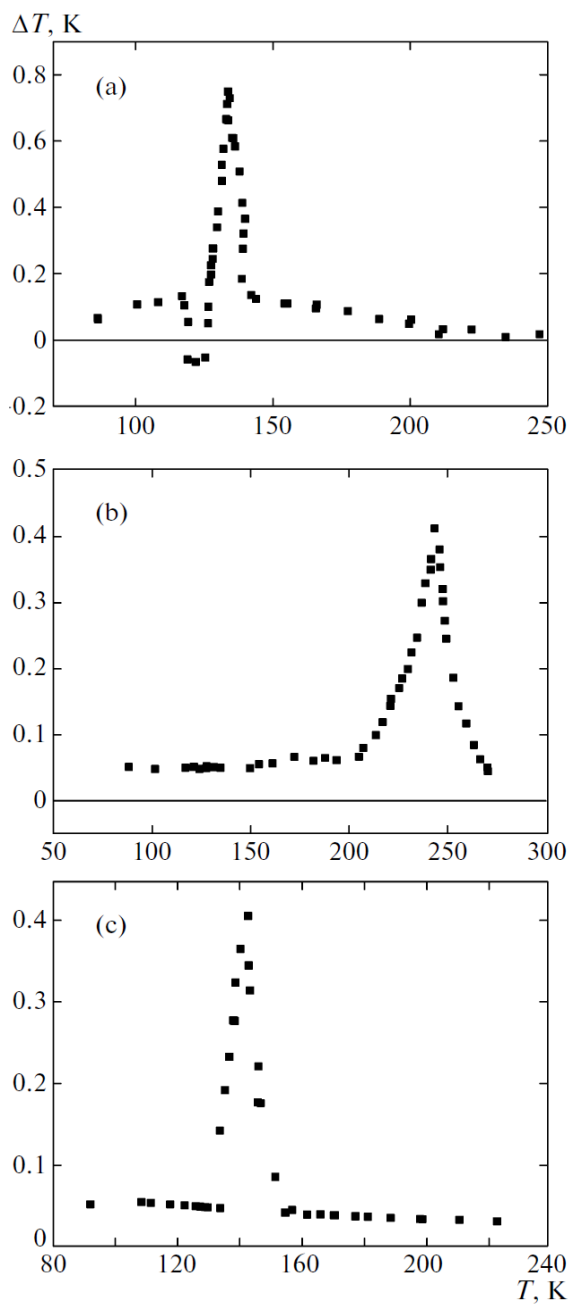

Figure 6. $\mathrm{La}_{1-\mathrm{x}} \mathrm{S} \mathrm{r}_{x} \mathrm{MnO}_{3}$ composition. Temperature dependences of $\mathrm{MCE}$ in a magnetic field of $14.2 \mathrm{kOe}$ for single-crystal samples (a) A (cooled in air) and (b) $O$ (cooled in an oxygen atmosphere) and (c) for polycrystalline sample $C$.

In this time the negative MCE is absent on the $\Delta T_{e x}(T)$ curves of $\mathrm{O}$ - and $\mathrm{C}$-samples. Figure 7 shows the $\Delta T_{e x}(H)$ dependence at $T_{\max }=143 \mathrm{~K}$ for sample $\mathrm{A}$, and a similar $\Delta T_{e x}(H)$ dependence at $T=T_{\max }$ was observed in samples $\mathrm{O}$ and $\mathrm{C}$.

As is seen from Eq. (1), which describes MCE in the paraprocess range, MCE is positive upon a break in the $\mathrm{F}$ ordering and is negative upon a break in the AF ordering. Obviously, the maximum on the $\Delta T_{e x}(T)$ curves of samples $\mathrm{A}$ and $\mathrm{C}$ are related to the sharp decrease in the magnetization of $\mathrm{F}$ clusters near their Curie point, and a break in the AF ordering in A-type AF clusters causes the minimum in the $\Delta T_{e x}(T)$ curve of single crystal sample $\mathrm{A}$. It is possible that this break in the AF order also continues at the maximum in the $\Delta T_{e x}(T)$ curve and decreases it; that is, the contribution of the break in the AF order in A-typeAF clusters to the total MCE ofthe, sample decrease its magnitude and slightly shifts the maximum toward high temperatures. It is apparent that the AF contribution decreases the total MCE of C-sample too; This decreasing is smaller in A-sample than in $\mathrm{C}$-samples. As a result, the maximum 
onthe $\Delta T_{e x}(T)$ curve is detected slightly above the Curie temperature rather than at this temperature, as in most MCE materials [27].

It clearly demonstrates that technology of preparation of samples may be modified the relation between $\mathrm{F}$ and AF portions of sample.

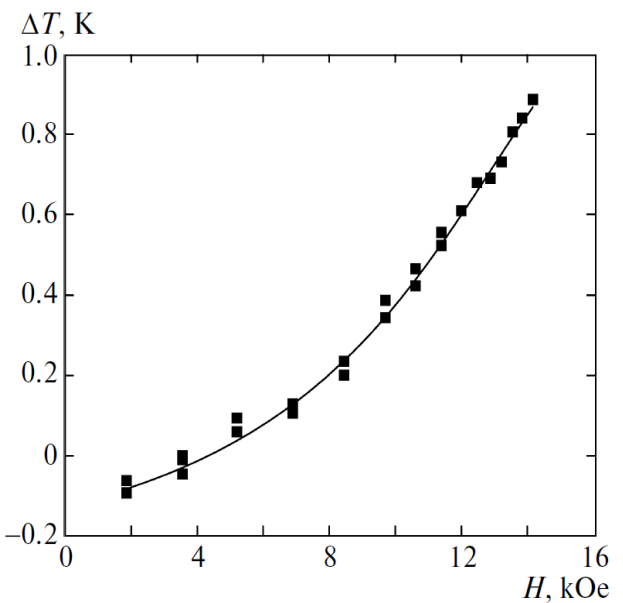

Figure 7. The dependence of magnetocaloric effect from magnetic field $H$ at $\mathrm{T}=143 \mathrm{~K}$ of the $\mathrm{Sm}_{0.55} \mathrm{Sr}_{0.45} \mathrm{MnO}_{3}$ single crystal annealed in air atmosphere (A-sample).

It is obvious that higher magnitudes of MCE can be achieved in $\mathrm{S}_{0.55} \mathrm{Sr}_{0.45} \mathrm{MnO}_{3}$ in higher fields. This is indicatedby the fact that the maximum magnitude of MCE increases with $H$ in the magnetic field range up to 14.2 kOe (Figure 7), with the rate of increase at $H>8 \mathrm{kOe}$ being higher than at $H<8 \mathrm{kOe}$. As is seen from Figure. $6 \mathrm{~b}$, this composition exhibits a jumplike increase in the magnetization in a certain critical field range $H c_{1}<H<$ $H c_{2}$, which increases linearly with thetemperature. This jumplike increase is related to thetransition of CE type AF clusters into the $\mathrm{F}$ state. It is seen from a comparison of Figures 7 and $4 \mathrm{c}$, themagnetic field range $8 \mathrm{kOe}<H<$ $14.2 \mathrm{kOe}$, in which MCE increases faster than at $H<8$ $\mathrm{kOe}$, is located in the critical field range $H c_{1}<H<H c_{2}$ and $H c_{2}>14.2 \mathrm{kOe}$. By this is meant that the $\Delta T_{e x}(H)$ curve is far from saturation and in the stronger magnetic field MCE may be great.

We emphasize that the maximum on $\Delta T_{e x}(T)$ curve of O-sample, cooled in oxygen atmosphere, is disposed at $T_{\max }=243 \mathrm{~K}$, which is significantly higher than $T_{C}$, and isvery close to $T=240 \mathrm{~K}$, at which the break of AF of CE-type ordering occur. Apparently the annealing of sample in oxygen closes the broken connection Mn-V-Mn (here $\mathrm{V}$ is vacancy of oxygen) and in doing so stabilizes AF phase of CE-type which become a dominating in sample. It should be noted that MCE is positive near $243 \mathrm{~K}$ whence it follows that it is connected with the $\mathrm{F}$ order break. It is possible that magnetic field, applied to sample at $\Delta T$-measurement, transfers $\mathrm{AF}$ phase of CE-type in $\mathrm{F}$ state and in $T_{N}$ both are broken.

With the spontaneous magnetizations of all samples,

we calculated MCE by Eqs. (1) and (2) using the specific heat borrowed from [30]. The maxima of calculated values of MCE and those measured by the direct method are given in the table 1 , which also contains the Curie temperatures, the paramagnetic Curie points,andthe temperatures of the MCE maxima $\left(T_{\max }\right)$ or minima $\left(T_{\min }\right)$. The calculated values of MCE are seen to be higher than those measured by the direct method by a factor of 9 . Therefore, MCE in the $\mathrm{Sm}_{0.5+} \mathrm{Sr}_{0.5-x} \mathrm{MnO}_{3}(0.05 \leq x \leq$ 0.10 ) compounds should only be determined by the direct method, and the magnitude of the effect depends strongly on the sample preparation conditions.

\section{Magnetocaloric Effect in $\mathrm{PrBaMn}_{2} \mathrm{O}_{7}$}

In $\mathrm{PrBaMn}_{2} \mathrm{O}_{6}$ manganite with the layer crystalline structure $\mathrm{Pr}$ and $\mathrm{Ba}$ ions are crystallographic ordered. Its magnetic properties strongly depend on the degree of this ordering. Recent studies of neutron and electron diffraction and the magnetic susceptibility of $\mathrm{PrBaMn}_{2} \mathrm{O}_{6}$ [11] have shown that the compound with the degree of this crystallographic ordering $96 \%$ has two magnetic phase transitions: paramagnetic-F at $T_{C}=303 \mathrm{~K}$ and $\mathrm{F}-\mathrm{AF}$ at $T_{N}=252 \mathrm{~K}$. With the decreasing of the crystallographic ordering degree the quantity of frustrated connections increase and the spin glass state occurs in the fully disordered composition.

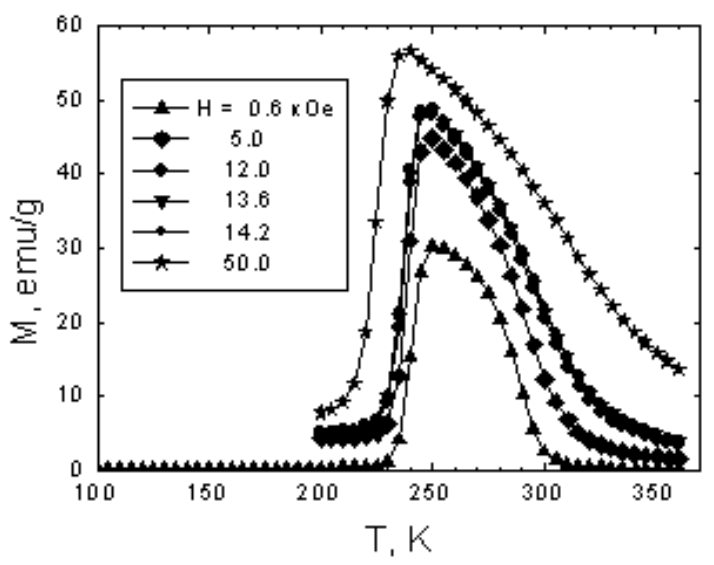

Figure 8. The temperature dependence of the magnetization of the $\mathrm{PrBaMn}{ }_{2} \mathrm{O}_{6}$ polycrystalline sample in some magnetic fields.

In our work the sample was studied in which the degree of crystallographic orderingreaches of $96 \%$. Figure 8 shows the temperature dependence of the magnetizationin the several magnetic fields of this sample. $T_{C}$, equal to $295 \mathrm{~K}$, was determined as temperature of maximum derivative $d M / d T$, in the $M(T)$ curve in a magnetic field of $14,2 \mathrm{kOe}$,It is magnetic field in which MCE was measured. As shows from Figure 8 the sharp drop of magnetization is observed at the Neel temperature $T_{N}=231 \mathrm{~K}$.Small difference of the TC and TNvalues received by we and in [30] is connected with difference of magnetic fields in which they were measured and the technology of samples. The temperature dependence of paramagnetic susceptibility obeys the Curie-Weiss law with a paramagnetic Curie temperature $\Theta=297.5 \mathrm{~K}$ which is close to the Curie temperature.

Phase with spontaneous magnetization obtains the understated magnetic moment. So magnetic moment calcu- 
lated from the maximum magnetization in the $\mathrm{M}(\mathrm{T})$ curvesat $14,2 \mathrm{kOe}$ and $50 \mathrm{kOe}$ equal to $4,9 \mu \mathrm{B}$. and 4,2 $\mu \mathrm{B}$ per chemicalformula respectively (Figure 8). Chemical formula contain 3 magnetic ions: $\mathrm{Mn} 3+(4 \mu \mathrm{B})$, $\mathrm{Mn} 4+(3 \mu \mathrm{B})$ and $\operatorname{Pr} 3+(3 \mu \mathrm{B})$. Evidently magnetic moment, received from experiment, is smaller than the one at $\mathrm{F}$ ordering the all magnetic ions. This is evidenced by the presence of nanoregions with the frustrated connections disposed in phase with spontaneous magnetization.

Figure 9 shows the temperature dependence of MCE.of this composition in a magnetic field of 14,2 kOe. The curve $\Delta \operatorname{Tex}(\mathrm{T})$ has two extremes: the broad maximum covered $\mathrm{TC}=295 \mathrm{~K}$ and the sharp minimum at $\mathrm{T}=234 \mathrm{~K}$ which is close to $\mathrm{TN}=231 \mathrm{~K}$. The magnitudes of MCE at both the maximum and minimum are low: it reaches 0.13 $\mathrm{K}$ at the maximum and $-0.2 \mathrm{~K}$ at the minimum. As is seen from the table 1, $\Delta \mathrm{Tth}$ calculated by Eq. (2) upon the transition from the antiferromagnetic to the ferromagnetic state is $-5.9 \mathrm{~K}$. Its absolute value is much higher than at the minimum. It is obvious that the low values of MCE at both the maximum and minimum are caused by the fact that ferromagnetic interactions are present in the antiferromagnetic phase and antiferromagnetic interactions exist in the spontaneous magnetization phase.

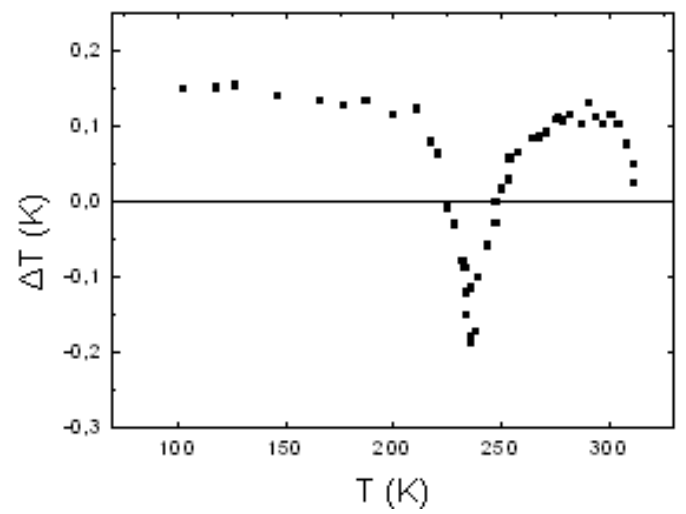

Figure 9. The temperature dependence of the magnetocaloric effect in magnetic field 14,2 kOe ofthe PrBaMn2O6 polycrystalline sample.

\section{Conclusions}

Using a direct method, we studied the MCE in La1xSrxMnO3, Sm0.55Sr0.45MnO3, andPrBaMn2O6 compounds, which represent three manganite classes, namely, La1 - xAxMnO3 $(\mathrm{A}=\mathrm{Ca}, \mathrm{Sr}, \mathrm{Ba}), \mathrm{Sm0.5}+\mathrm{xS} 0.5-$ $\mathrm{xMnO} 3(0.05 \leq \mathrm{x} \leq 0.10)$, and layered ReBaMn2O6 manganite. We measured the magnetization of these compositions and calculated the MCE related to the change in the magnetic part of entropy induced by a break in the spontaneous magnetization at the Curie temperature in the firsttwo compositions and by the transition from an $\mathrm{AF}$ into an F state at the Néel temperature of the last composition. The calculated value of MCE was found to be several times higher than the absolute value of MCE measured by a direct method. This difference was shown to be related to the presence of AF regions in the manganites with a predominant $\mathrm{F}$ order and the presence of $\mathrm{F}$ regions in the manganites with a predominant AF order. On the other hand, the low values of MCE in the manganites indicate the existence of a magnetically heterogeneous F-AF state in them. The presence of a colossal magnetoresistance and a giant volume magnetostriction $[16,20,32,33]$ points to that fact that this magnetically heterogeneous state is caused by a strong s- $d$ exchange and consists of an insulating AF phase and an $\mathrm{F}$ phase accumulating charge carriers [18].

It is known [27] that a high value of MCE in metals, e.g., gadolinium, requires a high purity of a metal. Impurities introduced AF bonds decreasing MCE.

\section{Acknowledgments}

We thank A.M. Balbashov, A.R. Kaul', and V.S. Kalitka for the preparation and analysis of the samples.

\section{References}

[1] [X.X. Zhang, J. Tajada, Y. Xin ,G.F. Sun, K.W. Wong, and X. Bahigas. "Magnetocaloric effect inLa0.67Ca0.33MnO $\delta$ and $\mathrm{La} 0.60 \mathrm{Y} 0.07 \mathrm{Ca} 0.33 \mathrm{MnO} \delta$ bulk materials." Appl. Phys. Lett., vol.69, pp. 3596-3598, December 1996.

[2] Z.B. Guo, Y.W. Du, J.S. Zhu, H. Huang, W.P. Ding, and D. Feng. "Large magnetic entropy change in perovskite-type manganese." Phys. Rev. Lett., vol. 78, pp. 1142-1145, March 1997.

[3] W. Chen, W. Zhong, D. Houn, D. Hou, W. Ding, Y. Du, and Yan O. Structure and magnetocaloric properties in perovskitelike La1-xNaxMnOz powders."Chin. Phys. Lett., vol.15, pp. 134-136, February 1998.

[4] X. Bohigwas, J. Tejada, E. Del Barco, X.X. Zhang, and M. Sales. "Tunable magnetocaloric effect in ceramic perovskites." Appl. Phys. Lett., vol. 73, pp.390-392, January 1998.

[5] V.K. Pecharsky, K.A. Gschneidner."Magnetocaloric effect and magnetic refrigeration.” J. Magn. Magn. Mater., vol. 200, pp. 44-46, October 1999.

[6] X. Bohigas, J. Tejada, M.L Marinez-Sarrion, S. Tripp, and R. Black. "Magnetic and calorimetric measurement on the magnetocaloric effect in $\mathrm{La} 0.6 \mathrm{Ca} 0.4 \mathrm{MnO} 3$. .J. Magn. Magn. Mater., vol. 208, pp. 85-92, January 2000.

[7] R.V. Demin, L.I. Koroleva. "Influence of the magnetic two-phase state on the magnetocaloric effect in manganites." Phys. Lett. A, vol. 317, pp. 140-143, July2003.

[8] A.I. Abramovich, A.V. Michurin, O.Yu. Gorbenko, and A.R. Kaul."Giant magnetocaloric effect near Curie point in $\mathrm{Sm} 0 / 6 \mathrm{Sr} 0.4 \mathrm{MnO} 3$ manganites." Phys. Solid State, vol. 43, pp. 687-689, April 2001.

[9] A.G. Gamzatov, A.M. Aliev, A.B. Batdalov, Sh.B. Abdulvagidov, O.V. Mel'nikov, and O.Yu. Gorbenko. "Magnetocaloric effect in Ag-doped of lanthanummanganite." Tech. Phys. Lett., vol. 32, pp. 471-477, August 2006.

[10] Yi Lu, Nai-li Di, Guang-jin Wang, Qing-an Li, and Zhaohua Cheng. "Low-field-induced magnetic entropy change in single-crystal Nd0.47Sr0.53MnO3"J. Phys.: Condens. Matter., vol. 16, pp. L243-246,April 2004.

[11] M. Phan, H. Peng, and S. Yu."Large magnetocaloric effect in single crystal Pr0.63Sr0.37MnO3.’J.Appl. Phys., vol.97, 
pp. 10M306 (3 p.), October 2005.

[12] I.K. Kamilov, A.G. Gamzatov, A.M. Aliev, A.B. Batdalov, A.L. Aliverdiev, Sh.B. Abdulvagidov, O.V. Mel'nikov, O.Yu. Gorbenko, R. Kaul. "Magnetocaloric effect in La1xAgyMnO3 $(\mathrm{y} \leq \mathrm{x})$ : direct and indirect measurements."J. Phys. D: Appl. Phys., vol. 40, pp. 4413-4417, August, 2007.

[13] I.R. Kamilov, A.G. Gamzatov, A.B. Batdalov, A.S. Mankevich, and I.E. Korsakov. "Thermal theat and magnetocaloric properties ofLa1-xKxMnO3 manganites." Phys. Solid State, vol. 52, pp. 728-734, April 2010.

[14] A.G. Gamzatov, A.B. Batdalov,A.M. Aliev, L.N. Khanov, H. Ahmad, H. Salamati, and P. Kameli. "Magnetocaloric effect in Pr1-xAgxMnO3manganite.”JETP Lett. vol.91, pp. 369-373. April 2010.

[15] D.M. Zashchirinskii, L.I. Koroleva, A.S. Morozov, and Yu.Y. Dolzhenkova. "Magnetocaloric effect in Sm0.55Sr0.45MnO3." Phys. Solid State, vol. 53, pp. 316320,February 2011.

[16] R. V. Demin, L. I. Koroleva, R. Szymszak, and H. Szymszak, "Experimental evidence for a magnetic two-phase state in manganites." JETP Lett., vol. 75, pp. 331-335, August 2002 .

[17] X. Moya, L.E. Hueso, F. Maccherozzi, A.I. Tovstolytkin, D.I. Podyalovskii, C. Ducati, L.C. Phillips, M. Ghidini, O. Hovorka, A. Berger, M.E. Vickers, E. Defay, S.S. Dhesi, and D. Mathur. "Giant and reversible magnetocaloric effects in $\mathrm{La} 0.7 \mathrm{Ca} 0.3 \mathrm{MnO} 3$ films due to strain". Nature Materials, vol. 12, pp. 52-58, January 2013.

[18] E.L. Nagaev, Colossal Magnetoresistance and Phase Separation in Magnetic Semiconductors, Imperial College Press: Singapore 2002, pp.1- 461.

[19] E. Dagotto, T. Hotta, and A. Moreo, "Colossal magnetoresistant materials: the key role of phase separation." Phys. Rep., vol. 344, pp. 1-153, January 2001.

[20] L.I. Koroleva. Magnetic semiconductors. Publishing ofphysics department of MSU: Moscow 2003, pp. 1-315.

[21] L.I. Koroleva, R. Szymczak, "Ferromagneticantiferromagnetic state in manganites.”J. Phys. Chem. Sol., vol. 64, pp. 1565-1568, November2003.

[22] G.L. Liu, J.S. Zhou, J.B. Goodenough, "Interplay between charge, orbilal, and magnetic ordering in La1-xSrxMnO3." Phys. Rev., vol. B64, pp.1-7, January 2001.

[23] B.V. Znamenskii, I.G. Fakidov. "Magnetization and mag- netocaloric effect of $\mathrm{Cu}+22,8$ at.\% Mn polycrystalline alloy.” Fiz. Met. Metalloved., vol. 13, pp. 312-314, February 1962.

[24] M. Viret, H. Glattli, C. Fermon, A.M. Leon-Guevara, andA. Revcolevschi. "Magnetic Coherenceabove the Curie point in ferromagnetic LaSrMnO manganites." Europhys Lett., vol. 42, pp. 301-303, April 1998.

[25] V.A. Ivashin, J. Deisenhofer, H.-A. Krug von Nidda, A. Loidl, A.A. Mukhin, A.M. Balbashov, andV. Eremin. „ESR study in lightly doped La1-xSrxMnO3." Phys. Rev., vol.B61, pp. 6213-6219, March2000.

[26] A. Machida, Y. Moritomo, A. Nakamura Temperature evolution ofthe 1,5 eV absorption band indoped manganites. Formation of Jahn Teller clusters."Phys. Rev., vol. B58, pp.12540-12542,November 1998.

[27] A.M. Tishin "Magnetocaloric effect in the vicinity of phase transitions." In: Handbook of magnetic materials, vol.12,ed. K.H.J. Buschop, Elsevier Sci. B.V., 1999, pp. 395-524.

[28] V. V. Runov, D. Yu. Chernyshov, A. I. Kurbakov,M. K. Runova, V. A. Trunov, and A. Okorokov "Mesoscopic magnetic heterogeneities in the lowtemperature phase and structure of Sm1-xSrxMnO3 (x $<0,5)$ perovskite." JETP,vol. 118, pp. 1174-1187, November 2000.

[29] C. Martin, A. Maignan, M. Hervieu, and B. Raveau, "Magnetic phase diagrams of $\mathrm{L} 1-\mathrm{xAxMnO} 3$ manganites (L = Pr, Sm; A = Ca, Sr).”Phys. Rev., vol. B60, pp. 12191 (8 p.), November 1999.

[30] A. M. Aliev, Sh. B. Abdulvagidov, A. B. Batdalov,I. K. Kamilov, O. Yu. Gorbenko, and V. A.Amelichev. "Specific heat and electrical resistivity of $\mathrm{Sm} 0.5 \mathrm{Sr} 0.5 \mathrm{MnO} 3$ in field up to 26 kOe.”JETP Lett., vol. 72, pp. 464-468, May 2000.

[31] T. Nakajima, H. Yoshizawa, Y. Ueda, A-site radomness effect on structural and physical properties ofBa-based perovskite manganites.“J. Phys.Soc. Japan, vol. 73, pp. 22832291, August 2004.

[32] A.I. Abramovich, L.I. Koroleva, A.V. Michurin. "Peculiarities of the magnetic, galvanomagnetic, elastic, and magne-

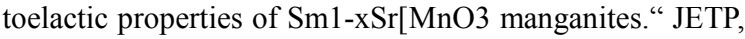
vol. 95, pp. 917-926, May 2002.

[33] L.I. Koroleva, R.V. Demin, A.V. Kozlov, D.M. Zashchirinskii and Ya.M. Mukovskii. Relation between giant volume magnetostric- tion, colossal magnetoresistance, and crystal lattice softening in manganites La1-xAxMnO3 $(\mathrm{A}=$ Ca, Ag, Ba, Sr). JETP, vol. 104, pp. 76-86, January 2007. 